\title{
The Spin of the Black Hole in the Soft X-Ray Transient A0620-00
}

\section{Citation}

Gou, Lijun, Jeffrey E. McClintock, James F. Steiner, Ramesh Narayan, Andrew G. Cantrell, Charles D. Bailyn, and Jerome A. Orosz. 2010. "The Spin of the Black Hole in the Soft X-Ray Transient A0620-00." The Astrophysical Journal 718 (2) (July 9): L122-L126. doi:10.1088/2041-8205/718/2/l122..

\section{Published Version}

doi:10.1088/2041-8205/718/2/L122

\section{Permanent link}

http://nrs.harvard.edu/urn-3:HUL.InstRepos:26526404

\section{Terms of Use}

This article was downloaded from Harvard University's DASH repository, and is made available under the terms and conditions applicable to Other Posted Material, as set forth at http:// nrs.harvard.edu/urn-3:HUL.InstRepos:dash.current.terms-of-use\#LAA

\section{Share Your Story}

The Harvard community has made this article openly available.

Please share how this access benefits you. Submit a story.

\section{Accessibility}




\title{
THE SPIN OF THE BLACK HOLE IN THE SOFT X-RAY TRANSIENT A0620-00
}

\author{
Lijun Gou ${ }^{1}$, Jeffrey E. McClintock ${ }^{1}$, James F. Steiner ${ }^{1}$, Ramesh Narayan ${ }^{1}$, Andrew G. Cantrell ${ }^{2}$, Charles D. Bailyn ${ }^{2}$, \\ AND JEROME A. OROSZ ${ }^{3}$ \\ ${ }^{1}$ Harvard-Smithsonian Center for Astrophysics, Cambridge, MA 02138, USA \\ 2 Department of Astronomy, Yale University, P.O. Box 208101, New Haven, CT 06520, USA \\ ${ }^{3}$ Department of Astronomy, San Diego State University, 5500 Campanile Drive, San Diego, CA 92182, USA \\ Received 2010 February 17; accepted 2010 June 29; published 2010 July 9
}

\begin{abstract}
During its year-long outburst in 1975-76, the transient source A0620-00 reached an intensity of $50 \mathrm{Crab}$, an all-time record for any X-ray binary. The source has been quiescent since then. We have recently determined accurate values for the black hole (BH) mass, orbital inclination angle, and distance. Building on these results, we have measured the radius of the inner edge of the accretion disk around the BH primary by fitting its thermal continuum spectrum to our version of the relativistic Novikov-Thorne thin-disk model. We have thereby estimated the spin of the BH. Although our spin estimate depends on a single high-quality spectrum, which was obtained in 1975 by $O S O-8$, we are confident of our result because of the consistent values of the inner-disk radius that we have obtained for hundreds of observations of other sources: H1743-322, XTE J1550-564, and notably LMC X-3. We have determined the dimensionless spin parameter of the BH to be $a_{*}=0.12 \pm 0.19$, with $a_{*}<0.49$ and $a_{*}>-0.59$ at the $3 \sigma$ level of confidence. This result takes into account all sources of observational and model-parameter uncertainties. Despite the low spin, the intensity and properties of the radio counterpart, both in outburst and quiescence, attest to the presence of a strong jet. If jets are driven by BH spin, then current models indicate that jet power should be a steeply increasing function of $a_{*}$. Consequently, the low spin of A0620-00 suggests that its jet may be disk driven.
\end{abstract}

Key words: accretion, accretion disks - black hole physics - stars: individual (A0620-00) - X-rays: binaries

Online-only material: color figure

\section{INTRODUCTION}

A0620-00 is the prototype soft X-ray transient (SXT), which is an eruptive type of X-ray binary. For several days in 1975, the flux at Earth from this source was greater than the combined total flux of all the other Galactic X-ray binaries, including Sco X-1. A decade later, a dynamical study of its quiescent optical counterpart (V616 Mon) led to the discovery of the first black hole (BH) primary in an SXT (McClintock \& Remillard 1986). A0620-00 is one of the eight, similar short-period BH SXTs $\left(P_{\text {orb }}<12 \mathrm{hr}\right.$; Remillard \& McClintock 2006). In the optical band, it is the best-studied of these systems because its counterpart is bright $\left(V_{\text {quiescent }}=18.3\right)$ and close: $D=1.06 \pm 0.12 \mathrm{kpc}$ (Cantrell et al. 2010).

The radial velocity amplitude of the secondary star is firmly established and has now been determined to the remarkable precision of $0.1 \%$ (Neilsen et al. 2008). However, a reliable estimate of the $\mathrm{BH}$ mass $M$, which depends on a robust determination of the orbital inclination angle $i$, has remained elusive. Several measurements of $i$ have been made by modeling the ellipsoidal variability of the secondary, but they have been inconsistent (Neilsen et al. 2008). The determination of $i$ is complicated by a variable and phase-dependent component of light from the accretion disk. Recently, however, a comprehensive analysis of all of the available light curve data (32 data sets spanning 30 years) points to consistent values of inclination and $\mathrm{BH}$ mass: $i=51.0 \pm 0.9$ and $M=6.61 \pm 0.25 M_{\odot}$ (Cantrell et al. 2010).

Our group has published spin estimates for five stellar-mass BHs (Shafee et al. 2006; McClintock et al. 2006; Liu et al. 2008; Gou et al. 2009). Meanwhile, the spins of several stellar-mass $\mathrm{BHs}$ have also been obtained by modeling the profile of the $\mathrm{Fe} \mathrm{K}$ line (see Miller et al. 2009 and references therein). The spins we find are all quite high, with values of the spin parameter $a_{*}$ in the range 0.7 to $>0.98$. The dimensionless quantity $a_{*} \equiv c J / G M^{2}$ with $\left|a_{*}\right| \leqslant 1$, where $M$ and $J$ are, respectively, the BH mass and angular momentum (Shapiro \& Teukolsky 1983). We use the $\mathrm{X}$-ray continuum-fitting method, which was pioneered by Zhang et al. (1997). Our spin estimates are based on our version of the Novikov-Thorne thin accretion disk model (Li et al. 2005) and an advanced treatment of spectral hardening (Davis et al. 2005; Davis \& Hubeny 2006). We only consider spectra that contain a dominant thermal component (Steiner et al. 2009a) and for which the Eddington-scaled bolometric disk luminosity is moderate, $l \equiv L_{\mathrm{bol}}\left(a_{*}, \dot{M}\right) / L_{\mathrm{Edd}}<0.3$ (McClintock et al. 2006).

For the continuum-fitting method to succeed, it is essential to have accurate values of $M, i$, and $D$ (e.g., Gou et al. 2009), such as those reported above for A0620-00. Herein, we use these input data and the only suitable, extant X-ray spectrum of the source in order to estimate the spin of the BH primary. Although our spin measurement is based on a single spectrum, we have considerable confidence in our result for the following two reasons. (1) In our earlier work, we have found that our measurements of spin, or equivalently the dimensionless radius of the innermost stable circular orbit $\left.r_{\mathrm{isco}} \equiv R_{\mathrm{ISCO}} /\left(G M / c^{2}\right)\right)$, for a particular source are remarkably consistent over years or decades (e.g., Gou et al. 2009; Steiner et al. 2009a, 2010). Most compelling is our recent study of the persistent source LMC X-3: Hundreds of observations obtained over a span of 26 years by eight different missions give values of $r_{\text {isco }}$, and hence $a_{*}$, that are consistent within a few percent (Steiner et al. 2010). One important conclusion from this study is that a single high-quality spectrum is a good proxy for a large collection of spectra. (2) The $O S O-8$ spectrum in question is a very high quality spectrum for the determination of spin via the continuum-fitting method: it is a remarkably pure thermal spectrum that is almost completely 
free of the effects of Comptonization (Section 3), and it was obtained using a stable, advanced proportional-counter detector that was the forerunner of the RXTE Proportional Counter Array detectors.

\section{DATA SELECTION AND REDUCTION}

A0620-00 was observed using the Goddard Cosmic Xray Spectroscopy Experiment (GCXSE) on board OSO-8 (Serlemitsos et al. 1976, 1977). The detector employed, namely, the C Detector, is a sealed multiwire xenon-methane proportional counter with a net effective area of $237 \mathrm{~cm}^{2}$, which is fitted with a 5.1 (FWHM) circular collimator. The observation commenced on 1975 September 29 at 11:58 UT and continued for 3.0 days during which the intensity of the source was $\approx 11$ Crab (Matilsky et al. 1976). The average collimator-corrected raw count rate was $\approx 1650$ counts $\mathrm{s}^{-1}$ and varied by $\approx 20 \%$ over the course of the observations. The gain and detector resolution $(18 \%$ at $6 \mathrm{keV})$ were determined using an on-board ${ }^{241} \mathrm{Am}$ source, and the energy calibration is good to a precision of $\sim 1 \%$ (Mushotzky et al. 1978).

We first ran the FTOOL osofindfast to determine the "good observing days" and then downloaded the appropriate raw pulseheight analyzer (PHA) data. These data had already been filtered to eliminate times of high background during passage through the South Atlantic Anomaly and times of Earth occultation. Attending only to the data from Detector $\mathrm{C}$, we ran the tool osopha to extract the 63 channel spectrum (spanning 2-60 keV), and we added a $2 \%$ systematic error to all the channels; osopha automatically corrected the counting rates for dead time by the factor of 0.704 . The effective source exposure time for the observation is $21.3 \mathrm{ks}$. We then used the tools osofindfast and osopha to extract and examine background spectra from several locations. Our results are insensitive to the choice of the background region, and we finally settled on a region centered at $l=201.9$ and $b=-28.2$ and an effective background observation time of $3.35 \mathrm{ks}$. We computed a correction to the response of the detector by comparing the power-law spectrum of Toor \& Seward (1974), our standard reference spectrum (McClintock et al. 2006), to the parameters derived by analyzing a spectrum of the Crab that was obtained just 12 days prior to the observation of A0620-00. Following a new correction procedure (Steiner et al. 2010), we computed a Toor \& Seward normalization coefficient $f_{\mathrm{TS}}=0.792$ and a slope difference $\Delta \Gamma_{\mathrm{TS}}=-0.148$, where $f_{\mathrm{TS}}$ is the ratio of the observed normalization to that of Toor \& Seward and $\Delta \Gamma_{\mathrm{TS}}$ is the difference between the observed value of the photon index and that of the reference spectrum. This correction is applied in all the analysis work below via a custom XSPEC multiplicative model.

\section{ANALYSIS AND RESULTS}

Preliminary, nonrelativistic analysis. All of the data analysis and model fitting throughout this paper were performed using XSPEC version 12.6 (Arnaud 1996). As in our earlier work (e.g., Gou et al. 2009), we first make an assessment of the data by analyzing it with the nonrelativistic disk model DISKBB. For modeling the weak Compton component of emission we use our convolution model SIMPL (Steiner et al. 2009b), which far outperforms the standard power-law model (POWERLAW) with its troublesome divergence at low energies (Yao et al. 2005; Steiner et al. 2009a). The parameters of SIMPL and POWERLAW are similar; their principal parameter, the photon index $\Gamma$ is identical. However, the normalization parameter for SIMPL is the scattering fraction-the fraction $f_{\mathrm{SC}}$ of the seed photons that are scattered into the power-law tail-rather than the photon flux.

Thus, the model we first employ is TBABS(SIMPL $\otimes$ DISKBB), where TBABS is a widely used model of low-energy absorption (Wilms et al. 2000). We fitted the data over the energy range 2.2-17 keV (Mushotzky et al. 1978). Because of the detector's limited low-energy response, we are unable to fit for the hydrogen column density $N_{\mathrm{H}}$, which we estimate from published values of the reddening. Based on the five refereed papers known to us, we find $0.25<E(B-V)<0.45$ with a most frequently cited value of 0.35 (Wu et al. 1983). We adopt the value $E(B-V)=0.35 \pm 0.05$. Assuming $A_{\mathrm{V}} / E(B-V)=3.1$ and $N_{\mathrm{H}} / A_{\mathrm{V}}=2.0 \times 10^{21} \mathrm{mag}^{-1} \mathrm{~cm}^{-2}$ (Predehl \& Schmitt 1995; Güver \& Özel 2009), we obtain our estimate of the column density: $N_{\mathrm{H}}=2.2 \times 10^{21} \mathrm{~cm}^{-2}$.

The fit to the PHA spectrum is $\operatorname{good}\left(\chi_{v}^{2}=1.03\right)$, and the temperature is precisely determined: $k T=0.700 \pm 0.004 \mathrm{keV}$. Moreover, the parameters of the Comptonized emission are well determined: $\Gamma=3.81 \pm 0.37$ and $f_{\mathrm{SC}}=0.008 \pm 0.003$. The best-fit value of the scattering fraction is low, $0.8 \%$ (compare Gou et al. 2009 and Steiner et al. 2009a), and it is even lower for the relativistic model $(0.6 \%$; see below). Thus, this spectrum is thermal dominant in the extreme (Remillard \& McClintock 2006). Because the Compton component is faint, the fitted values of both $k T$ and $a_{*}$ depend very weakly on how one models this nonthermal emission.

We are fortunate that in the $O S O-8$ GCXSE software instructions, precisely the same spectrum of A0620-00 that we consider was used to illustrate how one reduces and analyzes $O S O-8$ data $^{4}$. The model employed in this example is PHABS(DISKBB+POWERLAW), and the fixed column density is $\approx 35 \%$ higher, $N_{\mathrm{H}}=3.0 \times 10^{21} \mathrm{~cm}^{-2}$. Using this model, we obtain precisely the same temperature reported in the example, namely, $k T=0.70 \mathrm{keV}$, which confirms the correctness of our reduction/analysis procedures.

Relativistic analysis. We now turn to the analysis of the data using our fully relativistic accretion disk model KERRBB2, which includes self-irradiation of the disk ("returning radiation") and limb darkening (Li et al. 2005). The effects of spectral hardening are incorporated into the basic model KERRBB via a pair of look-up tables for the hardening factor $f$ corresponding to two representative values of the viscosity parameter: $\alpha=0.01$ and 0.1 (see Gou et al. 2009). The entries in this table were computed using a second relativistic disk model BHSPEC (Davis et al. 2005). We refer to the model KERRBB plus this table/subroutine as KERRBB2. The model KERRBB2 has just two fit parameters, namely, the $\mathrm{BH}$ spin parameter $a_{*}$ and the mass accretion rate $\dot{M}$. For further details see McClintock et al. (2006).

We now analyze the data in exactly the same manner as before, except that we replace DISKBB by KERRBB2: TBABS(SIMPL $\otimes$ KERRBB2). We fix the column density at $N_{\mathrm{H}}=$ $2.2 \times 10^{21} \mathrm{~cm}^{-2}$, and we have four free parameters: the dimensionless spin parameter $a_{*}$, the mass accretion rate $\dot{M}$, the photon index of the high-energy component $\Gamma$, and the scattering fraction $f_{\mathrm{SC}}$. We fitted the spectrum with the input parameters fixed at their baseline values (see footnote to Table 1). The normalization was fixed at unity (as appropriate when $M, i$, and $D$ are held fixed). We included the effects of limb darkening $($ lflag $=1)$ and returning radiation (rflag $=1)$, and we set the

\footnotetext{
4 http://heasarc.gsfc.nasa.gov/docs/oso8/software/oso8_example3.html
} 
Table 1

KERRBB2 Fit Results for A0620-00a

\begin{tabular}{cccccrr}
\hline \hline & $N_{\mathrm{H}^{\mathrm{b}}}$ & $\Gamma$ & $f_{\mathrm{SC}}$ & $a_{*}$ & $\dot{M}^{\mathrm{c}}$ \\
\hline 1 & 2.2 & $3.53 \pm 0.38$ & $0.0062 \pm 0.0030$ & $0.135 \pm 0.029$ & $1.62 \pm 0.06$ & 0.109 \\
2 & $1.9^{\mathrm{e}}$ & $3.51 \pm 0.38$ & $0.0060 \pm 0.0029$ & $0.141 \pm 0.028$ & $1.60 \pm 0.06$ & 0.109 \\
3 & $2.5^{\mathrm{e}}$ & $3.55 \pm 0.39$ & $0.0064 \pm 0.0032$ & $0.126 \pm 0.029$ & $1.63 \pm 0.06$ & 0.109 \\
4 & 2.2 & $3.91^{\mathrm{f}}$ & $0.0099 \pm 0.0005$ & $0.116 \pm 0.019$ & $1.65 \pm 0.04$ & 0.74 \\
5 & 2.2 & $3.15^{\mathrm{f}}$ & $0.0038 \pm 0.0002$ & $0.155 \pm 0.025$ & $0.58 \pm 0.05$ & 0.108 \\
\hline
\end{tabular}

Notes.

${ }^{\mathrm{a}} M=6.61 M_{\odot}, i=51.0, D=1.06 \mathrm{kpc}$, and $\alpha=0.01$.

${ }^{\mathrm{b}}$ Column density is in units of $10^{21} \mathrm{~cm}^{-2}$.

${ }^{\mathrm{c}}$ Mass accretion rate is in units of $10^{18} \mathrm{~g} \mathrm{~s}^{-1}$.

${ }^{\mathrm{d}} l \equiv L_{\mathrm{bol}}\left(a_{*}, \dot{M}\right) / L_{\mathrm{EDD}}($ see Section 1$)$.

e Corresponds to $E(B-V)=0.35 \pm 0.05$ (see Section 3).

${ }^{\mathrm{f}}$ Corresponds to $\Gamma=3.53 \pm 0.38$ from row 1 .

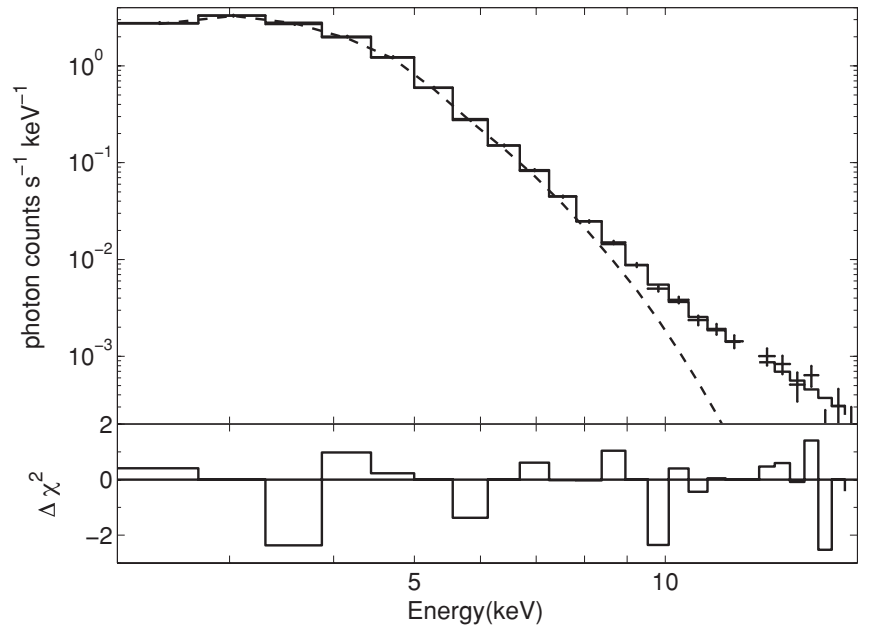

Figure 1. Unfolded $O S O-8 \mathrm{X}$-ray spectrum of A0620-00. Top: the histogram shows the model fitted to the data with the dashed line representing the thermal component. Bottom: the residuals (data minus model in units of $\chi_{v}^{2}$ ). The data for the bin centered at $E \approx 12.7 \mathrm{keV}$ were corrupted and are ignored (see footnote 1 in the text).

torque at the inner boundary of the disk to zero $(\eta=0)$. The fitted results are presented in the first row of Table 1. Compared to the nonrelativistic fit, the fit here is even better $\left(\chi_{v}^{2}=0.74\right)$. The luminosity is moderate $(l \approx 0.1)$ and easily meets our selection criterion $l<0.3$ (Section 1). Of prime interest, we find a low and precise value of the spin parameter, $a_{*}=0.135 \pm 0.029$.

The unfolded photon spectrum and fit residuals are shown in Figure 1. The best-fit value of the scattered fraction is very low, only $0.6 \%$ (Table 1 ). The second and third rows of Table 1 show that the uncertainty in $N_{\mathrm{H}}$ has a quite modest effect on the value of the spin parameter, shifting the best-fit value of $a_{*}$ by $<0.22 \sigma$. The last two rows of Table 1 further show that fixing the power-law index $\Gamma$ at $\pm 1 \sigma$ from its best-fit value of 3.53 also has a modest effect on $a_{*}$, shifting its best-fit value by at most $\approx 0.55 \sigma$. In fact, relative to the total uncertainty in $a_{*}$ these small shifts are still over 10 times smaller, i.e., $\lesssim 0.1 \sigma$, as we now show.

Comprehensive error analysis. The statistical uncertainty in $a_{*}$ is small, and other sources of error dominate. Ignoring for now uncertainties in the theoretical model (see Section 4), we consider the effects of uncertainties in (1) the input parameters $M, i$, and $D$, (2) the column density $N_{\mathrm{H}}$, (3) the viscosity parameter $\alpha$ (Davis et al. 2005), and (4) the metallicity Z. We first note that, as in the case of LMC X-1 (Gou et al. 2009), the uncertainty in the metallicity of the disk gas is negligible compared to the statistical error: we find that $a_{*}$ changes by only $0.34 \sigma$ when we vary the metallicity from our default value of $Z=1$ (solar) to $Z=0.1$.

In order to determine the error in $a_{*}$, we performed an analysis that considers at once the above items (1) and (2), i.e., the combined uncertainties in $M, i, D$, and $N_{\mathrm{H}}$. We fix the viscosity parameter to its baseline value, $\alpha=0.01$. In order to determine the error in $a_{*}$, we performed Monte Carlo simulations assuming that the uncertainties in the four parameters are normally and independently distributed. Specifically, we (1) generated 3000 parameter sets for $M, i, D$, and $N_{\mathrm{H}} ;(2)$ computed for each set the look-up table for the spectral hardening factor $f$ using the model BHSPEC; and (3) using these $f$-tables, obtained $a_{*}$ by fitting our model to the spectrum. The results for the 3000 simulation runs are shown in Figure 2. The uncertainty in $D$ (panel c) dominates the error in $a_{*}$, with the uncertainty in $M$ having less than half as much effect, while the uncertainties in $i$ and $N_{\mathrm{H}}$ are still smaller. The corresponding histogram for the spin displacements in $a_{*}$ about the most probable value is shown in Figure 3 by the light solid line.

The analysis above is for our baseline value of $\alpha=0.01$. Following precisely the same procedures, we also performed the Monte Carlo analysis for $\alpha=0.1$, and the result is the dashed-line histogram shown in Figure 3. The summation of these two histograms results in the large histogram (heavy solid line). The combined distribution, which corresponds to a total of 6000 simulations, has a median spin value of 0.12 and implies a $1 \sigma$ error of $(-0.19,+0.19)$. Thus, considering all significant observational and model-parameter uncertainties, we arrive at our final result for the spin of A0620-00: $a_{*}=0.12 \pm 0.19(1 \sigma)$. The corresponding radius of the inner disk, which is uncertain by $12 \%$, is just $7 \%$ less than the Schwarzschild value of $6 G M / c^{2}$.

\section{DISCUSSION}

Any measurement of $\mathrm{BH}$ spin is only as good as the theoretical model behind it. The continuum-fitting method assumes that the radial profile of the disk $L(R)$ is given by the analytical form derived by Novikov \& Thorne (1973, NT). Because any serious error in the NT model (e.g., the assumption of vanishing torque at the ISCO) will lead to large systematic errors in the derived $\mathrm{BH}$ spin values, we have mounted a major effort to scrutinize the NT model. In Shafee et al. (2008), we reported a three-dimensional GRMHD simulation of a thin disk $(H / R \sim$ 0.06) around a nonspinning $\mathrm{BH}$. We showed that the angular momentum profile matches the NT prediction to within $\sim 2 \%$, 

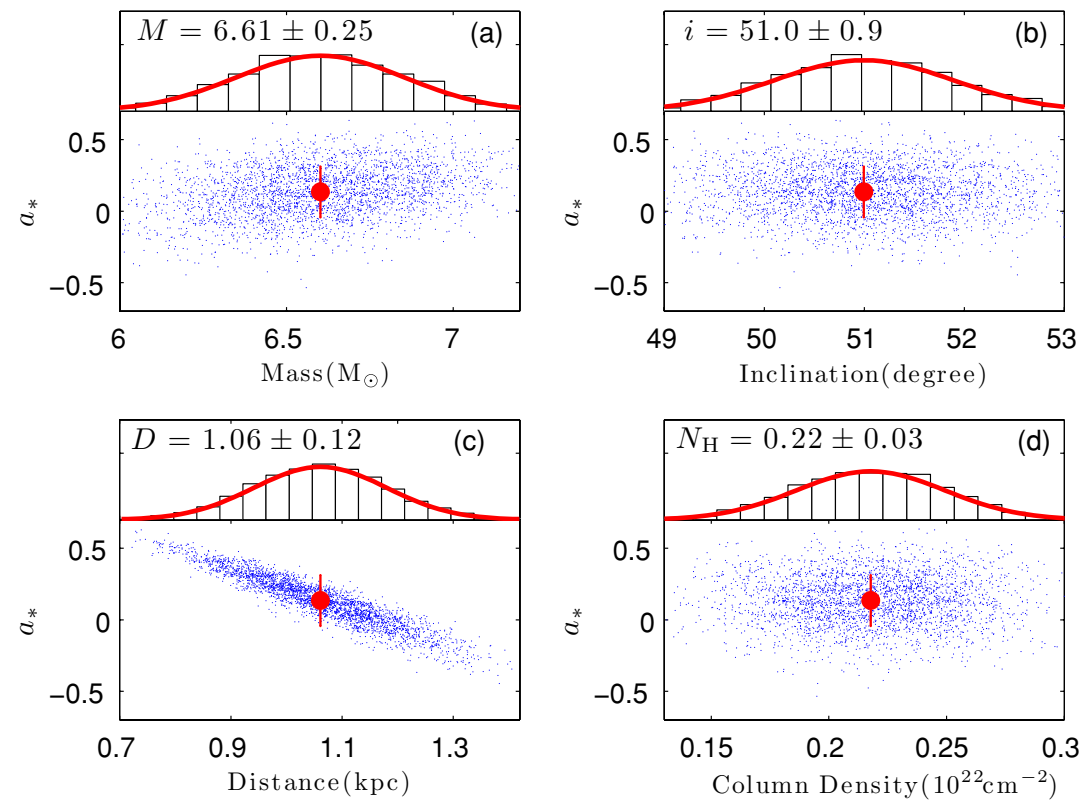

Figure 2. Effect on the spin parameter $a_{*}$ of varying $M, i, D$, and $N_{\mathrm{H}}$ for case $\alpha=0.01$. (a) The upper panel shows a normal distribution for the BH mass $M$ and the lower panel shows $a_{*}$ vs. mass $M$ for 3000 sets of parameters drawn at random. The central filled circle indicates our estimate of the spin $a_{* 0}=0.135_{-0.179}^{+0.178}$ obtained from these simulations. (b)-(d) Same as panel (a) except now for the parameters of inclination angle, distance, and column density, respectively.

(A color version of this figure is available in the online journal.)

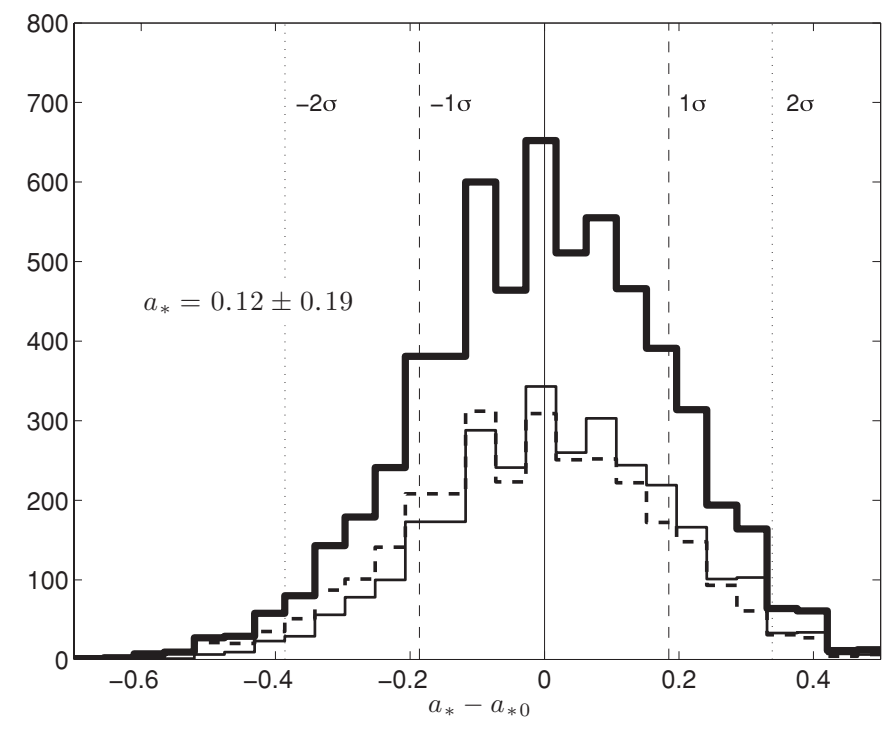

Figure 3. Combined error analysis that considers both of our fiducial values for the viscosity parameter. The thin solid line is for $\alpha=0.01$ and the dashed line is for $\alpha=0.1$. The sum of these two smaller histograms forms the large histogram. The vertical lines pertain to this latter histogram: the vertical solid line indicates the median value of the spin determined by these simulations: $a_{* 0}=0.12$; the two dashed lines enclose $68.27 \%(1 \sigma)$ of the spin values centered on the solid line and imply an observational uncertainty of $(-0.19,+0.19)$; the two dotted lines enclose $95.45 \%(2 \sigma)$ and imply an uncertainty of $(-0.39,+0.34)$. The upper and lower limits on the spin at the $99.73 \%(3 \sigma)$ level of confidence are respectively $a_{*}<0.49$ and $a_{*}>-0.59$.

indicating that any magnetic coupling across the ISCO is weak, and we estimated that the additional disk luminosity due to this weak coupling is only $\sim 4 \%$. Noble \& Krolik (2009) and Noble et al. (2010) have carried out simulations of similarly thin disks, but they conclude that deviations from the NT model are much larger, with the specific angular momentum deviating by up to $15 \%$. In a recent paper (Penna et al. 2010), we report simulations corresponding to a variety of disk thicknesses and BH spins. For thin disks with $H / R<0.07$ we agree with Shafee et al.'s conclusion that the NT model provides an accurate description of both the angular momentum profile and the luminosity (see Figures 14 and 15 in Penna et al.). We suggest that the contrary results obtained by Noble et al. are because (1) they used for the initial magnetic field in their simulations a topology with long-range radial coherence, which we argue is inappropriate for a thin disk, and (2) they included the contribution of the corona, even though the corona is generally believed not to participate in the optically thick thermal emission we model in the continuum-fitting method.

The inner X-ray-emitting portion of a thin accretion disk is presumably aligned with the spin axis of the $\mathrm{BH}$ (e.g., Lodato \& Pringle 2007). In determining the spin of A0620-00 and most sources, we assume that the BH's spin is closely aligned with the orbit vector; a misalignment of more than several degrees would significantly affect our results (e.g., see Figure 2(b)). There is presently no good evidence for significant misalignments despite two often-cited cases (see Section 2.2 in Narayan \& McClintock 2005). A recent population-synthesis simulation study indicates that the majority of $\mathrm{BH}$ binaries have relatively small misalignment angles $\left(\lesssim 10^{\circ}\right.$; Fragos et al. 2010). The approved NASA GEMS polarimetry mission, which is scheduled for a 2014 launch, is expected to soon provide the capability to directly determine the degree of alignment to an accuracy of a few degrees (Li et al. 2009; Kaaret et al. 2009).

Based on modeling the 1975-76 X-ray and optical light curves, Suleimanov et al. (2008) find a low and consistent value of spin, $a_{*} \sim 0.1$, for our mass of $M=6.6 M_{\odot}$ and for a fixed value of $f=1.7$ (see their Figure 7). However, for this model their fitted value of the viscosity parameter, $\alpha \approx 0.6$, is significantly higher than the higher fiducial value of $\alpha=0.1$ that we have adopted (Section 3; see discussion in Section 5.3 in Gou et al. 2009) or the values of $\alpha \sim 0.1-0.4$ based on the "best observational evidence" (King et al. 2007). We are unable to estimate $a_{*}$ for larger values of $\alpha$ because the required BHSPEC 
table models (Section 3) do not exist. However, it is clear (e.g., Figure 3) that larger values of $\alpha$ will decrease our estimate of $a_{*}$.

Recent GRMHD simulations by Fragile (2009) show that luminous, geometrically thick disks with $l \gtrsim 0.6$ (corresponding to $H / R \gtrsim 0.2$; McClintock et al. 2006) can falsely appear to host $\mathrm{BHs}$ with low spins if the inner disk is significantly tilted with respect to the $\mathrm{BH}$ spin axis. It is highly unlikely that this effect can explain the low spin of A0620-00 because the observed disk luminosity is low, $l \sim 0.1$ (Table 1 ), and the disk is correspondingly thin $H / R \sim 0.04$. In this case, as Fragile notes, one does not expect the inferred value of spin to be suppressed by the tilted-disk effect that he describes.

Despite its faint corona and low spin, A0620-00 was a bright transient radio source in outburst, decaying from $\sim 200 \mathrm{mJy}$ to $\lesssim 10 \mathrm{mJy}$ in the period from about 12 to 24 days following its discovery on 1975 August 3. A reanalysis of these data by Kuulkers et al. (1999) indicates multiple jet ejections of initially optically thick components. The authors find that the source was extended on arcsec scales, and they infer a relativistic expansion velocity. Even in quiescence, the radio source was detected at a level of $\sim 0.05 \mathrm{mJy}$, indicating the presence of a partially selfabsorbed synchrotron jet (Gallo et al. 2006). If jets are powered by $\mathrm{BH}$ spin, then jet power is likely to increase dramatically with increasing $a_{*}$ (McKinney 2005; Tchekhovskoy et al. 2010). The expected dependence is so steep that, for $a_{*}<0.4$, the jet receives more power from the accretion disk than from the $\mathrm{BH}$ (McKinney 2005). Therefore, given the low spin of A0620-00, it would appear that the jet inferred in this source was probably driven by the accretion disk, not the $\mathrm{BH}$. In closing, we note that a statistical study by Fender et al. (2010), although based on data of uneven quality, found no evidence that $\mathrm{BH}$ spin powers jets.

J.E.M. acknowledges support from NASA grant NNX08AJ $55 \mathrm{G}$ and the Smithsonian Endowment Funds. R.N. was supported in part by NSF grant AST-0805832 and NASA grant NNX08AH32G. We thank the following individuals for help in locating and utilizing the $O S O-8 \mathrm{X}$-ray spectrum: L. Angelini, G. Branduardi-Raymont, K. Pounds, J. Swank, and N. White. We thank J. McKinney, S. Murray, and an anonymous referee for helpful comments. This research has made exclusive use of data obtained from the High Energy Astrophysics Science Archive Research Center (HEASARC) at NASA/Goddard Space Flight Center.

\section{REFERENCES}

Arnaud, K. A. 1996, in ASP Conf. Ser., 101, Astronomical Data Analysis Software and Systems V, ed. G. H. Jacoby \& J. Barnes (San Francisco, CA: ASP), 17

Cantrell, A. G., et al. 2010, ApJ, 710, 1127

Davis, S. W., Blaes, O. M., Hubeny, I., \& Turner, N. J. 2005, ApJ, 621, 372

Davis, S. W., \& Hubeny, I. 2006, ApJS, 164, 530
Fender, R., Gallo, E., \& Russell, D. 2010, MNRAS, in press (arXiv: $1003.5516 \mathrm{v} 1$ )

Fragile, P. C. 2009, ApJ, 706, L246

Fragos, T., Tremmel, M., Rantsiou, E., \& Belczynski, K. 2010, ApJ, in press (arXiv:1001.1107v1)

Gallo, E., Fender, R. P., Miller-Jones, J. C. A., Merloni, A., Jonker, P. G., Heinz, S., Maccarone, T. J., \& van der Klis, M. 2006, MNRAS, 370, 1351

Gou, L. J., et al. 2009, ApJ, 701, 1076

Güver, T., \& Özel, F. 2009, MNRAS, 400, 2050

Kaaret, P., Swank, J., Jahoda, K., Kallman, T., \& The Gems Team 2009, in Conf Proc. Chandra's First Decade of Discovery, ed. S. Wolk, A. Fruscione, \& D. Swartz (Boston, MA: Harvard), 125

King, A. R., Pringle, J. E., \& Livio, M. 2007, MNRAS, 376, 1740

Kuulkers, E., Fender, R. P., Spencer, R. E., Davis, R. J., \& Morison, I. 1999, MNRAS, 306, 919

Li, L. X., Narayan, R., \& McClintock, J. E. 2009, ApJ, 691, 847

Li, L. X., Zimmerman, E. R., Narayan, R., \& McClintock, J. E. 2005, ApJS, 157,335

Liu, J. F., McClintock, J. E., Narayan, R., Davis, S. W., \& Orosz, J. A. 2008, ApJ, 679, L37

Lodato, G., \& Pringle, J. E. 2007, MNRAS, 381, 1287

Matilsky, T., et al. 1976, ApJ, 210, L127

McClintock, J. E., \& Remillard, R. A. 1986, ApJ, 308, 110

McClintock, J. E., Shafee, R., Narayan, R., Remillard, R. A., Davis, S. W., \& Li, L. 2006, ApJ, 652, 518

McKinney, J. C. 2005, ApJ, 630, L5

Miller, J. M., Reynolds, C. S., Fabian, A. C., Miniutti, G., \& Gallo, L. C. 2009, ApJ, 697, 900

Mushotzky, R. F., Serlemitsos, P. J., Boldt, E. A., Holt, S. S., \& Smith, B. W. 1978, ApJ, 225, 21

Narayan, R., \& McClintock, J. E. 2005, ApJ, 623, 1017

Neilsen, J., Steeghs, D., \& Vrtilek, S. D. 2008, MNRAS, 384, 849

Noble, S. C., \& Krolik, J. H. 2009, ApJ, 703, 964

Noble, S. C., Krolik, J. H., \& Hawley, J. F. 2010, ApJ, 711, 959

Novikov, I. D., \& Thorne, K. S. 1973, in Black Holes (Les Astres OccLus), ed. C. DeWitt \& B. DeWitt (New York: Gordon and Breach), 343

Penna, R. F., McKinney, J. C., Narayan, R., Tchekhovskoy, A., Shafee, R., \& McClintock, J. E. 2010, MNRAS, in press (arXiv:1003.0966v1)

Predehl, P., \& Schmitt, J. H. M. M. 1995, A\&A, 293, 889

Remillard, R. A., \& McClintock, J. E. 2006, ARA\&A, 44, 49

Serlemitsos, P. J., Becker, R. H., Boldt, E. A., Holt, S. S., Pravdo, S. H., Rothschild, R. E., \& Swank, J. H. 1976, NASA Special Publ., 589, 67

Serlemitsos, P. J., Smith, B. W., Boldt, E. A., Holt, S. S., \& Swank, J. H. 1977, ApJ, 211, L63

Shafee, R., McClintock, J. E., Narayan, R., Davis, S. W., Li, L., \& Remillard, R. A. 2006, ApJ, 636, L113

Shafee, R., McKinney, J. C., Narayan, R., Tchekhovskoy, A., Gammie, C. F., \& McClintock, J. E. 2008, ApJ, 687, L25

Shapiro, S. L., \& Teukolsky, S. A. 1983, Black Holes, White Dwarfs and Neutron Stars: The Physics of Compact Objects (New York: Wiley)

Steiner, J. F., McClintock, J. E., Remillard, R. A., Gou, L. J., Yamada, S., \& Narayan, R. 2010, ApJ, 718, L117

Steiner, J. F., McClintock, J. E., Remillard, R. A., Narayan, R., \& Gou, L. J. 2009a, ApJ, 701, L83

Steiner, J. F., Narayan, R., McClintock, J. E., \& Ebisawa, K. 2009b, PASP, 121, 1279

Suleimanov, V. F., Lipunova, G. V., \& Shakura, N. I. 2008, A\&A, 491, 267

Tchekhovskoy, A., Narayan, R., \& McKinney, J. C. 2010, ApJ, 711, 50

Toor, A., \& Seward, F. D. 1974, AJ, 79, 995

Wilms, J., Allen, A., \& McCray, R. 2000, ApJ, 542, 914

Wu, C., Panek, R. J., Holm, A. V., Schmitz, M., \& Swank, J. H. 1983, PASP, 95, 391

Yao, Y., Wang, Q. D., \& Zhang, S. N. 2005, MNRAS, 362, 229

Zhang, S. N., Cui, W., \& Chen, W. 1997, ApJ, 482, L155 